\title{
Imaging of the equatorial ionosphere
}

\author{
Massimo Materassi $\left({ }^{1}\right)$ and Cathryn N. Mitchell $\left({ }^{2}\right)$ \\ (1) Istituto dei Sistemi Complessi, CNR, Sesto Fiorentino (FI), Italy \\ $\left(^{2}\right)$ Department of Electrical and Electronic Engineering, University of Bath, U.K.
}

\begin{abstract}
The equatorial anomaly is the dominant structure in free electron concentration in the tropical ionosphere. Due to its edges (crests) which are characterised by steep latitudinal gradients in TEC and are temporally and spatially variable, it is one of the ionospheric regions most difficult to image with inversion methods. In this paper we reconstruct an International Reference Ionosphere model of the equatorial ionosphere by employing a grid of virtual ground GPS receivers and actual GPS satellite positions. The MIDAS algorithm, an inversion method for reconstructing the ionosphere as a linear composition of given empirical orthogonal functions, is used. Comparing the true model ionosphere with the resulting images a fine tuning of the basis functions (vertical profile contraints) in the inversion is realised.
\end{abstract}

Key words atmosphere-electromagnetic methods remote sensing - tomography - data processing GPS TEC

\section{Introduction}

The equatorial anomaly, with its crests either side of the geomagnetic equator, is one of the main features of the quiet ionosphere, and reproducing it correctly is an important challenge for any data analysis system for ionospheric imaging. Here we studied the possibility of producing Total Electron Content (TEC) maps and electron concentration sections using MIDAS (Multi Instrument Data Assimilation System), a linear inversion technique producing 3D time evolving ionospheric maps from merging of many different kinds of data. In particular, here we use simulated Global Positioning System (GPS) slant TEC data in order to reproduce a given model ionosphere.

Mailing address: Dr. Massimo Materassi, Istituto dei Sistemi Complessi, CNR, Via Madonna del Piano, 50019 Sesto Fiorentino (FI), Italy; e-mail: massimo.materassi@isc.cnr.it
The MIDAS technique is based on the use of orthogonal functions (EOFs), an idea suggested in Fremouw et al. (1992). The MIDAS inversions need a selection of functions to define the vertical profile of electron concentration. This kind of inversion problem is highly underdetermined in general (Fehmers, 1995) and this selection of profiles is the requested prior hypothesis. In MIDAS one assumes that the free electron density is a linear combination of a given set of three-dimensional EOFs. Here we apply an optimisation procedure to select the best fitting vertical profile EOFs for imaging the equatorial anomaly as represented by the International Reference Ionosphere (IRI) (Bilitza, 1990).

The inversions show that it is possible to obtain encouraging results. Once sufficient real GPS data through the equatorial ionosphere are made available, it is likely that accurate images of the equatorial ionosphere will be possible for geophysical studies and navigation applications.

\section{Method}

The MIDAS system reconstructs the free electron density as a piecewise constant 3D distribution, starting from collections of slant TEC 
data along ray paths crossing the region of interest (Mitchell and Spencer, 2003). The essential ingredient of MIDAS inversions is the use of Empirical Orthogonal Functions (Sirovich and Everson, 1992) along which the solution of the inverse problem is assumed to be linearly decomposable (Materassi, 2003). The most general form reads

$$
N_{\text {recon }}(\boldsymbol{r}, t)=\sum_{\alpha} X_{\alpha}(t) N_{\alpha}(\boldsymbol{r}, t) .
$$

Here $t$ is the time. The choice of the EOFs $N_{\alpha}(\boldsymbol{r}, t)$ is a key step for reconstructing the various ionospheric features. In (2.1) the coefficient $X_{\alpha}(t)$ is generally assumed to be linear with $t$ within one hour, that is the time interval used to collect GPS data to be inverted all together. Here, if

$$
X_{\alpha}(t)=A_{\alpha}+B_{\alpha}\left(t-t_{\text {start }}\right)
$$

holds, the unknowns of the tomographic inverse problem will be $A_{\alpha}$ and $B_{\alpha}$. In (2.2) $t_{\text {start }}$ is the starting time of the one hour inversion.

Simulated data are constructed with real GPS satellite positions and an ideal grid of simulated ground receivers. These receivers are placed on the vertices of the following net:

$$
\begin{aligned}
& \text { Latitude: }-25^{\circ} \div+5^{\circ} \text {, step: } 5^{\circ} \\
& \text { Longitude: }-80^{\circ} \div-40^{\circ} \text {, step: } 5^{\circ} \text {. }
\end{aligned}
$$

These virtual receiver locations are used in all our simulations, where the «true» ionosphere is assumed to be the IRI model. With this grid of virtual GPS receiver locations and real GPS satellite ephemerides, GPS slant TEC data are simulated and used for the MIDAS inversions. In fig. 1 the placement of the virtual receivers in (2.3) is illustrated, while the data coverage obtained with actual GPS satellite orbits and these receivers is illustrated in fig. 2. Here the rich geometry of the intersections of their ray paths with a thin shell at $400 \mathrm{~km}$ height is shown in order to give the idea of the situation of very good data coverage throughout the area of interest.

It is recognized that such good data coverage is not feasible when using real receivers. This study just aims to establish the influence

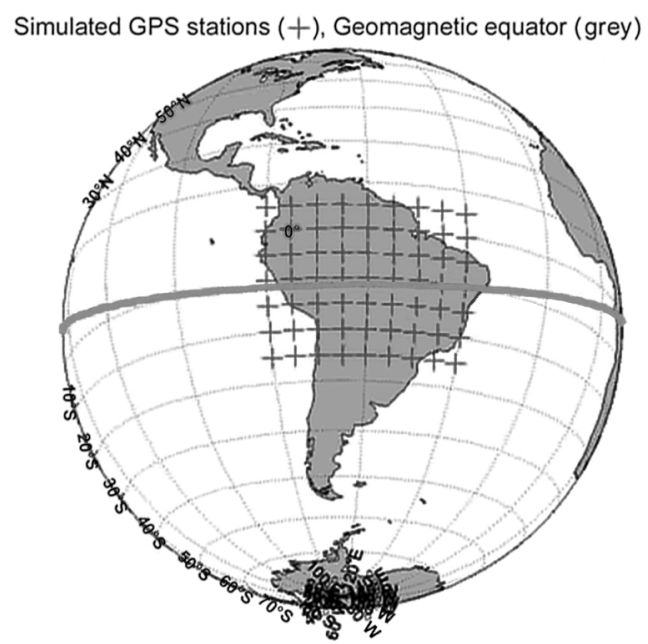

Fig. 1. The network of 56 virtual ground GPS receivers used in the present study. The grey curve represents the geomagnetic equator.

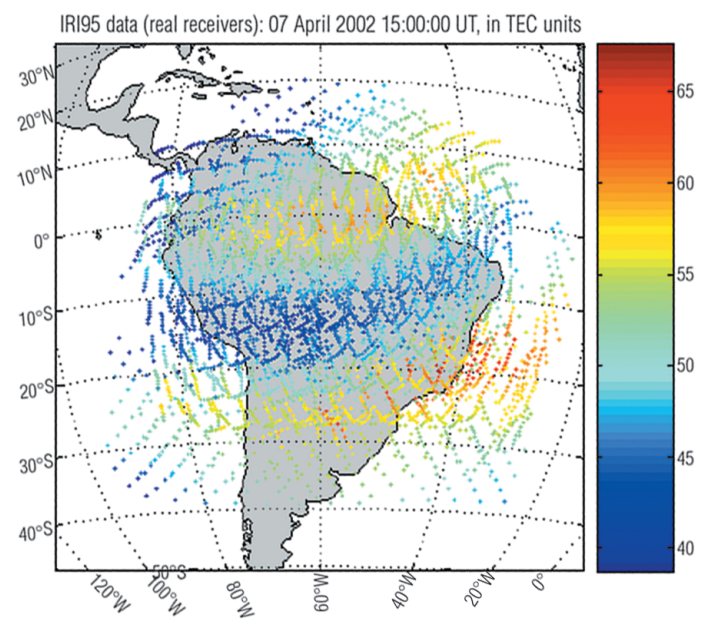

Fig. 2. Data coverage of the reconstruction region obtained by intersecting the ray paths with a shell at $400 \mathrm{~km}$ height. The colour code is associated with the slant TEC values.

of the choice of the EOFs when data geometry is very satisfactory. In forthcoming works, the same fine tuning will be studied for more realistic receiver configurations. 

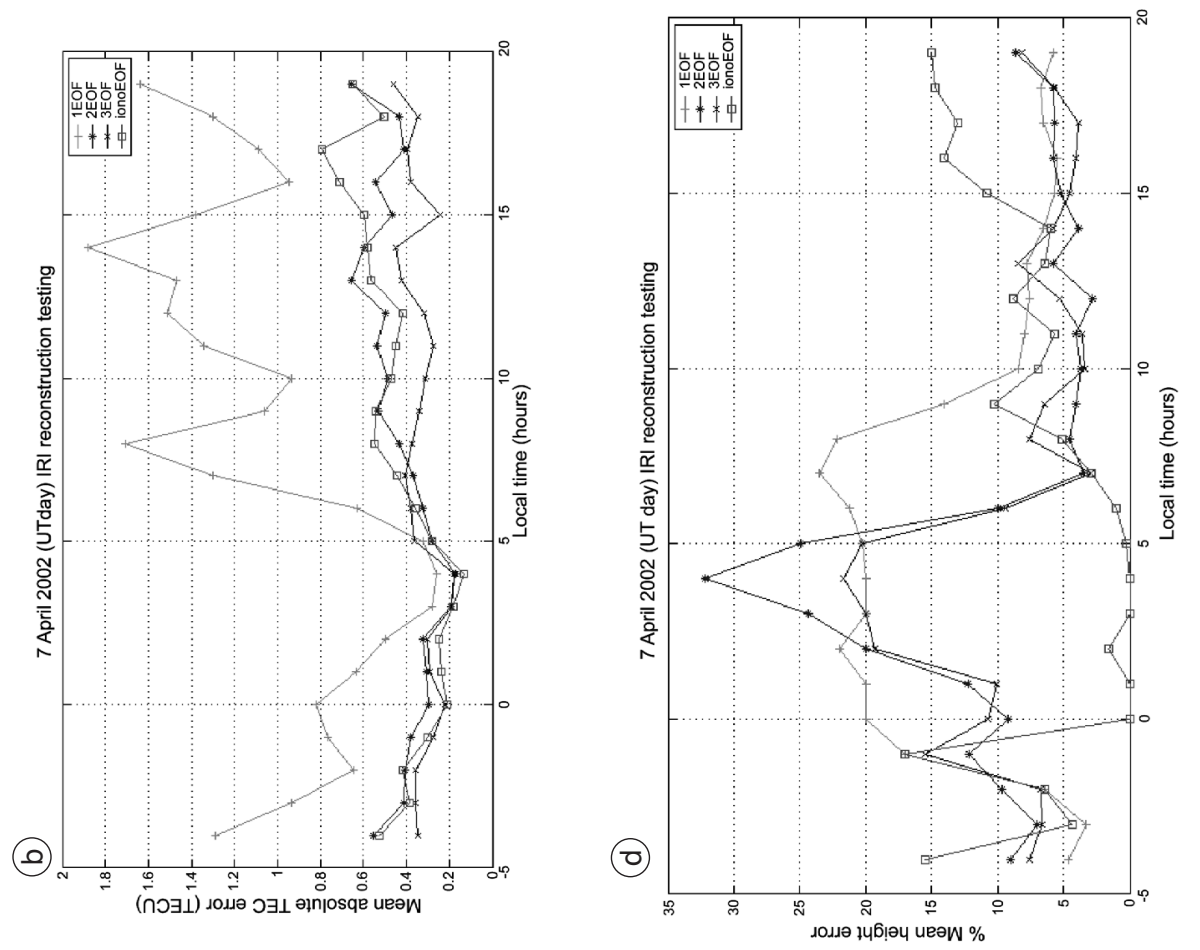

告
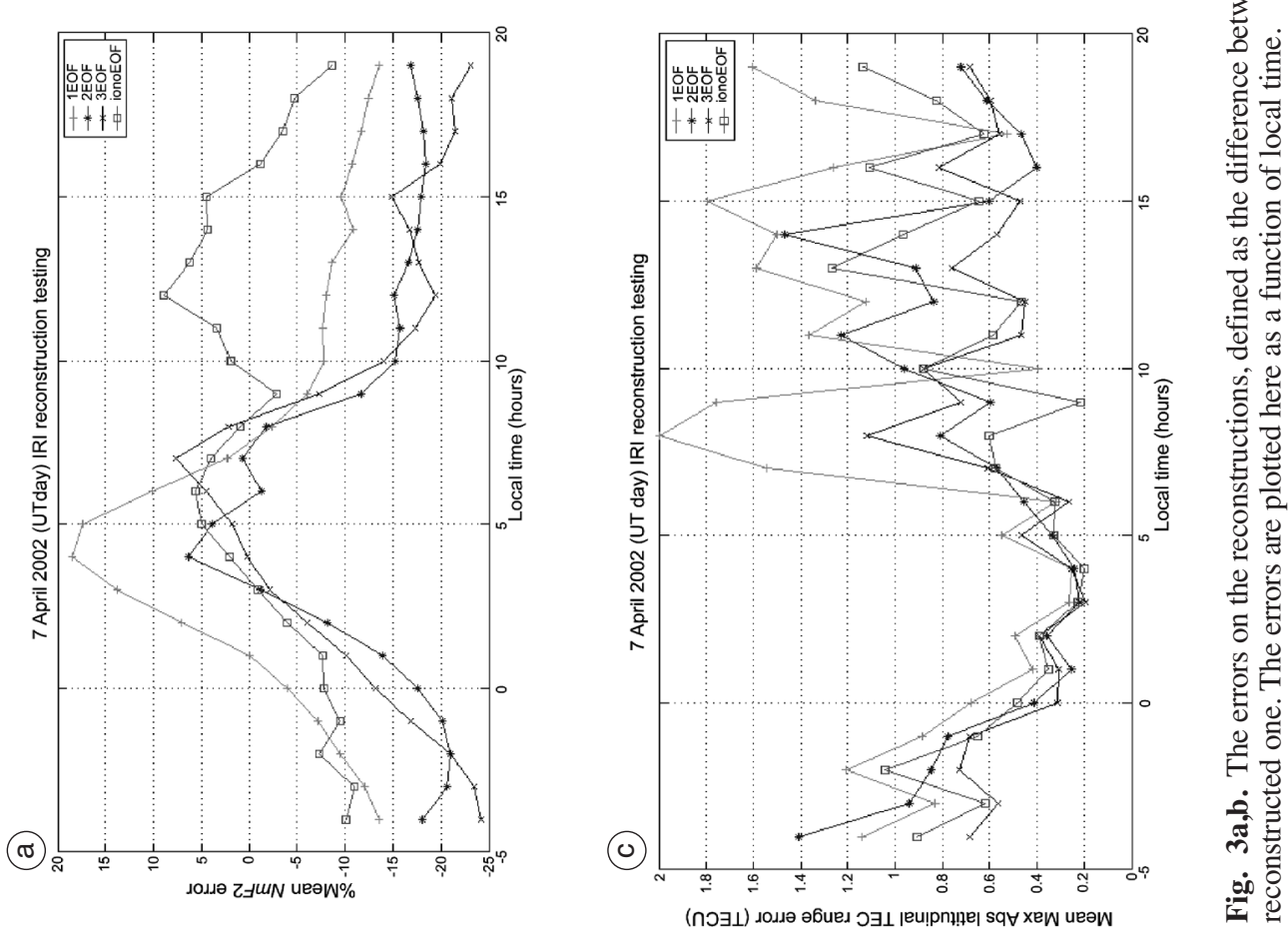


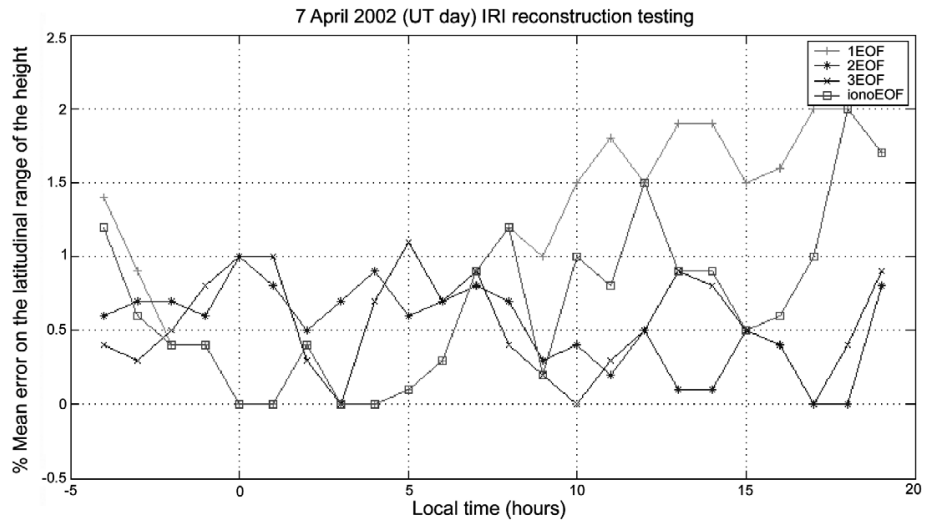

Fig. 3e. The errors on the reconstructions, defined as the difference between the quantity calculated for the model IRI ionosphere and for the MIDAS reconstructed one. The errors are plotted here as a function of local time.

Throughout this work, GPS data are simulated for an IRI model equatorial ionosphere using the network of virtual GPS receiver locations in (2.3) and actual satellite positions. The vertical behaviour of the EOFs is obtained from an assortment of Chapman profiles with physically reasonable peak heights and densities. The horizontal behaviour is constructed by modulating the vertical EOFs with 40 latitudinal harmonics and 4 longitudinal harmonics.

Different EOF bases were implemented for the reconstruction, distinguished by the different choices of the vertical functions. In order to get the three bases 1EOF, $2 \mathrm{EOF}$ and $3 \mathrm{EOF}$ one picks up respectively 1,2 and 3 orthonormal functions out of a collection of Chapman profiles defined by assorting sensible values of ionospheric parameters (peak heights and peak densities of the layers $F 1, F 2$ and $E$ ).

The basis IONO EOF is instead given by picking up two EOFs out of Chapman profiles, with peak heights and scale heights obtained from the simulated data that would be collected by nine ionosondes in the region of interest, observing the model IRI ionosphere.

The assessment of the quality of the reconstructions as obtained via different EOF bases is made by calculating some physical quantities $Q\left[N_{e}\right]$ depending on the free electron density. Such quantities are evaluated for the mod- el as $Q_{\text {mod }}=Q\left[N_{\text {IRI }}\right]$, and for the various inversions $Q_{\text {MIDAS }}=Q\left[N_{\text {recon }}\right]$. Comparing the discrepancies

$$
\Delta Q=Q_{\text {mod }}-Q_{\text {MIDAS }}
$$

between the $Q$ from the modeled and the reconstructed ionosphere, an assessment on the quality of the reconstruction is made. The quantities $Q$ calculated are the mean $N m F 2$ density, the mean vertical TEC, the mean TEC latitudinal range, the mean peak height and the mean peak height latitudinal range. The errors $\Delta Q$ used in the fine-tuning of the EOF basis, or their relative versions, are illustrated in fig. 3ae. They are plotted against the local time, in order to adapt the choice of the EOFs to the part of the day.

Figure 4 shows results from the worst choice, made of 1 vertical EOF modulated with the assortment of horizontal harmonics described before. The best-fitting EOF basis selected is instead a set made out of 2 vertical EOFs, 40 latitudinal harmonics and 4 longitudinal harmonics. The results obtained using it are shown in fig. 5. Better inversions are achieved when the EOF parameters are constructed from ionosonde data instead of being a general sensible set and the choice of 2 EOFs controlled by simulated ionosonde data reduces the errors. 
(b)
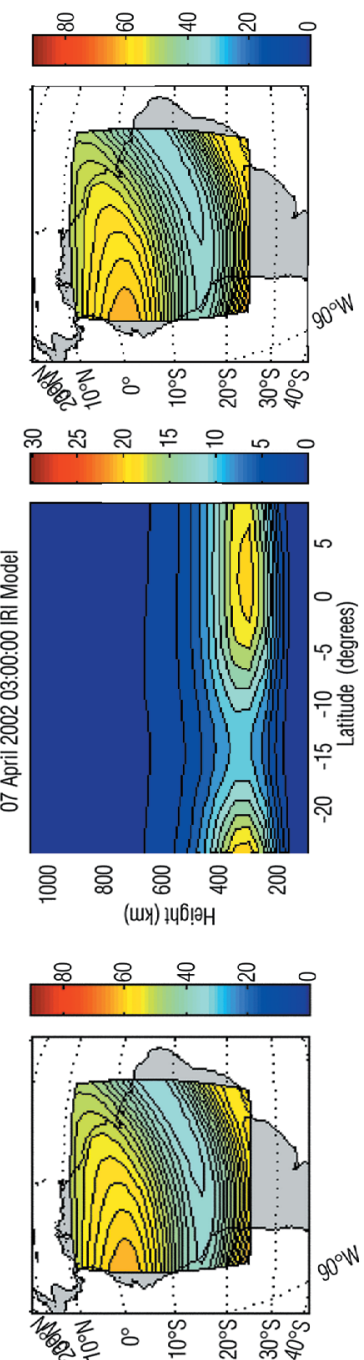

$\odot$

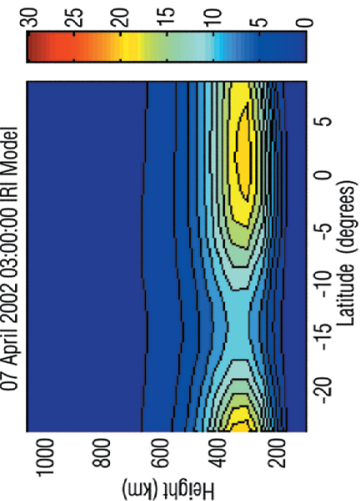

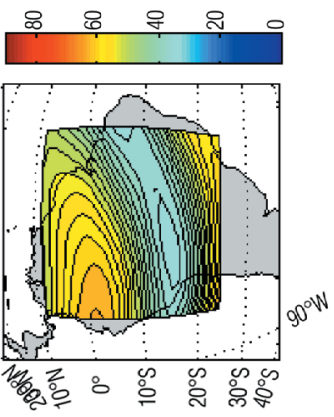
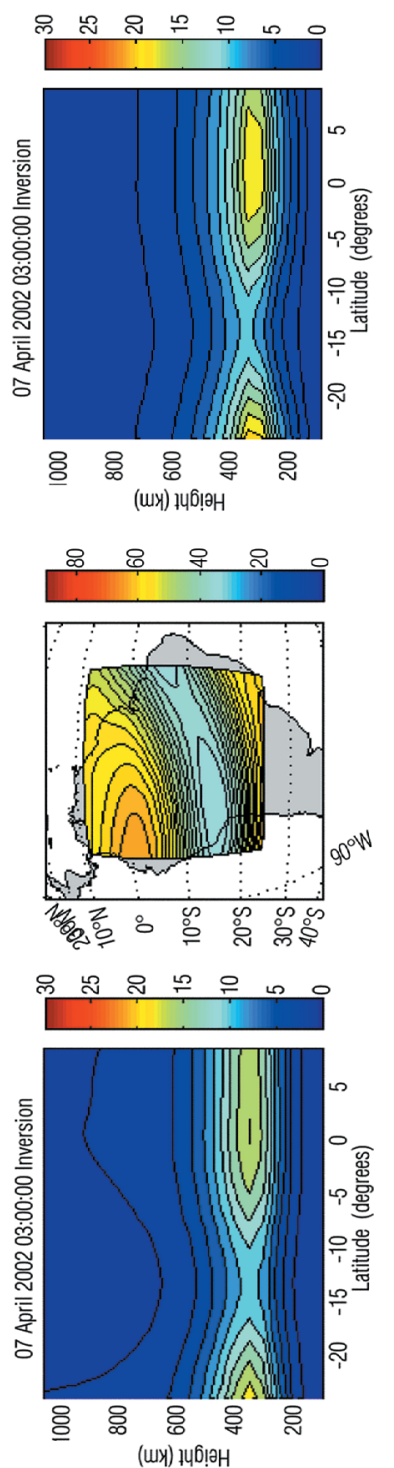

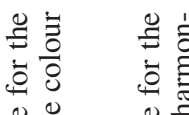

过

寉

ठำ

एक

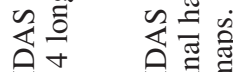

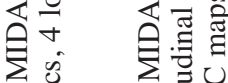

0.

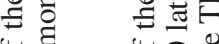

따유 늉워

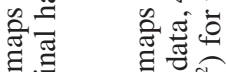

政

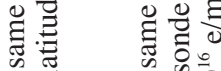

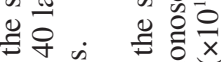

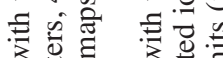

3 远焉

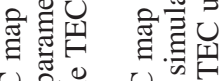

บ气 仓

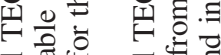

ฮี

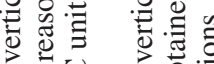

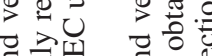

สี नี जี

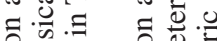

귤혀

के की कू क्जे

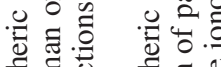

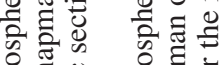

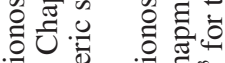

ब శٓ

융 चु

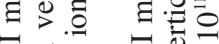

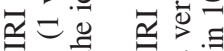

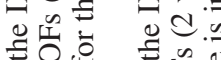

on

Eั है

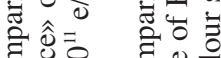

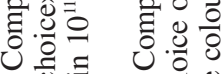

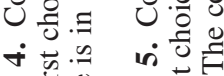

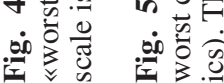




\section{Conclusions}

The possibility of reconstructing ionospheric maps of free electron density of an Equatorial IRI ionosphere by using the inversion technique MIDAS (Multi Instrumental Data Analysis System) has been shown.

The following points have been shown to be important for this region:

- The number of vertical EOFs is an important factor in accurately mapping the absolute values of TEC, at least two are needed.

- Horizontal TEC ranges are reproduced well by all our EOF choices.

- The formation of EOFs using information derived from simulated ionosonde parameters improves the determination of the peak heights and densities.

- The range in peak height (see fig. 3e) is not reproduced with one EOF, which by definition has a constant peak height. It is reproduced with more than one EOF.

Forthcoming work will be to reconstruct the actual equatorial electron densities by inverting real satellite data (from GPS as well as LEO satellites). The important issues identified in this simulation study will be taken into account and applied. In particular, it is very important to stress the great role that ionosonde data seem to be able to play in this kind of image reconstruction. Systematic studies on this point will be carried out in future works.

\section{Acknowledgements}

The authors acknowledge the use of the IRI model.

This work was funded by the U.K. EPSRC research council and supported by the authors' institutions. CNM is currently the recipient of an EPSRC Advanced Research Fellowship.

The authors are also deeply indebted to Luigi Ciraolo and Paolo Spalla of Istituto di Fisica Applicata «Nello Carrara» (IFAC-CNR) of Florence (Italy) for their useful criticism and for reading the manuscript.

\section{REFERENCES}

BiLITZA, D. (1990): International Reference Ionosphere 1990, NSSDC 90-22, Greenbelt, Maryland.

Fenmers, G. (1995): Tomography of the ionosphere, Degree Thesis at the Technische Universiteit Eindhoven, Eindhoven.

Fremouw, E.J., J.A. Secan and B.M. Howe (1992): Application of stochastic inverse-theory to ionospheric tomography, Radio Sci., 27 (3), 721-732.

MATERASSI, M. (2003): Ionospheric tomography, 3D and 4D imaging and data assimilation, invited paper at the 'Atmospheric Remote Sensing using Satellite Navigation Systems', Special Symposium of the URSI Joint Working Group FG, 13-15 October 2003, ASI Centro di Geodesia Spaziale «Giuseppe Colombo», Matera, Italy, 2003.

Mitchell, C.N. and P.S.J. SPENCER (2003): A three dimensional time-dependent algorithm for ionospheric imaging using GPS, Annal. Geophysics, 46 (4), 687-696.

Sirovich, L. and R. Everson (1992): Management and analysis of large scientific datasets, Int. J. Supercomputer Appl., 6, 50-68. 